Pacific Journal of Mathematics

THE PICARD THEOREM FOR MULTIANALYTIC FUNCTIONS 


\section{THE PICARD THEOREM FOR MULTIANALYTIC FUNCTIONS}

P. KRAJKIEWICZ

The class of multianalytic functions are defined. For this class the notions of essential and nonessential isolated singularities and of exceptional values are introduced. It is then shown that a multianalytic function has at most one exceptional value at an essential isolated singularity.

Suppose that $G$ is an arbitrary nonempty region of the finite complex plane $\Gamma$ and that $n \geqq 0$ is a nonnegative integer. A function $f: G \rightarrow \Gamma$ is said to be $(n+1)$-analytic on $G$ or simply polyanalytic on $G$ if and only if there exist $(n+1) \geqq 1$ functions $f_{k}$ analytic on $G$ for $k=0,1, \cdots, n$ such that

$$
f(z)=\sum_{k=0}^{n} \bar{z}^{k} f_{k}(z),
$$

for all $z$ in $G$, where $\bar{z}$ denotes the complex conjugate of $z$. A function $f$ is said to be $(n+1)$-entire or simply polyentire if and only if this function $f$ is $(n+1)$-analytic on $\Gamma$. A function $f$ is said to be bianalytic on $G$ if and only if this function $f$ is $(n+1)$-analytic on $G$ with $n=1$. Also a function $f$ is termed bientire if and only if this function $f$ is bianalytic on $\Gamma$.

Now let $f: G \rightarrow \Gamma$ be a function which is polyanalytic on $G$ and suppose that the function $f$ is represented on $G$ by equation (1). It is not hard to be persuaded that the functions $f_{k}$ in equation (1) are uniquely determined on $G$ by the function $f$. With this observation in mind the following definitions are not ambiguous. Let $z_{0}$ be an arbitrary complex number, finite or infinite. Then the point $z_{0}$ is said to be an isolated singularity of $f$ if and only if there is some neighborhood $N$ of $z_{0}$ such that $N-\left\{z_{0}\right\} \subseteq G$. The point $z_{0}$ is called an essential isolated singularity of $f$ or simply an essential singularity of $f$ if and only if the point $z_{0}$ is an isolated singularity of at least one of the functions $f_{k}$.

In [1], M. B. Balk derived the small Picard theorem for bientire functions by appealing to Picard's big theorem for analytic functions and the theory of quasi-normal families of analytic functions $[6, p$. 66]. Then in [2], the same author derived the big Picard theorem for bientire functions by applying similar methods. Later in [3], the same author succeeded in establishing Picard's big theorem for the larger class of polyentire functions by utilizing a theorem of Saxer [7], which generalizes the classical Schottky theorem. Finally in [4], a 
general version of Picard's big theorem was established for the still larger class of polyanalytic functions at an arbitrary isolated singularity, finite or infinite, by means of the Poisson-Jensen integral formula [5, p. 1] and quasi-normal families of analytic functions.

The results cited above invite some interesting speculation. The class of polyanalytic functions can be enlarged in various obvious ways. It is then natural to inquire as to whether a version of Picard's theorem is also valid for such a larger class of functions. In this paper we introduce such a larger class of functions which for the purposes of this paper are called multianalytic functions. We then establish a version of the big Picard theorem for the class of multianalytic functions at an arbitrary isolated singularity, finite or infinite. The above result seems to be noteworthy on two accounts. In our formulation of Picard's big theorem for multianalytic functions we have adopted a rather general definition of an essential isolated singularity of a multianalytic function and we have also employed a rather general notion of an exceptional value for a multianalytic function at an isolated singularity.

Now let $z_{0}$ be an arbitrary complex number, finite or infinite, and let $0<R<+\infty$. If $z_{0}$ is finite, then $A\left(z_{0}, R\right)$ will denote the set of all finite complex numbers $z$ such that $0<\left|z-z_{0}\right|<R$. If $z_{0}=\infty$, then $A\left(z_{0}, R\right)=A(\infty, R)$ will denote the set of all finite complex numbers $z$ such that $R<|z|<+\infty$. We also set $N\left(z_{0}, R\right)=A\left(z_{0}, R\right) \cup\left\{z_{0}\right\}$. Finally if $z_{0}$ is finite, then $C\left(z_{0}, R\right)$ will denote the circumference with center $z_{0}$ and radius $R$ which is oriented in the counterclockwise sense.

A function $f: A\left(z_{0}, R\right) \rightarrow \Gamma$ is said to be multianalytic on $A\left(z_{0}, R\right)$ if and only if there is some nonnegative integer $n \geqq 0$ and a sequence $\left\{f_{k}\right\}$ of functions analytic on $A\left(z_{0}, R\right)$ for all $k \geqq-n$ such that

$$
f(z)=\sum_{k=-n}^{+\infty}\left(\overline{z-z_{0}}\right)^{k} f_{k}(z),
$$

for all $z$ in $A\left(z_{0}, R\right)$. If $z_{0}=\infty$, then in the series in equation (2) it is understood that the term $\overline{\left(z-z_{0}\right)^{k}}$ is replaced by $1 / \bar{z}^{k}$ for each $k \geqq-n$. It is also understood that the series in equation (2) is almost uniformly convergent on $A\left(z_{0}, R\right)$, that is the series is uniformly convergent on every nonempty closed subset of $A\left(z_{0}, R\right)$. If $z_{0}$ is any complex number, finite or infinite, then a function $f$ is said to be multianalytic at $z_{0}$ if and only if there is some $R>0$ so that $f$ is multianalytic on $A\left(z_{0}, R\right)$.

Observe that if the functions $f_{k}$ in equation (2) are actually analytic in $N\left(z_{0}, R\right)$ for each $k \geqq-n$, then the series in equation (2) is almost uniformly absolutely convergent in $A\left(z_{0}, R\right)$.

We now wish to introduce a notion of an essential isolated singularity for multianalytic functions. If $f$ is multianalytic at $z_{0}$ and is 
represented on $A\left(z_{0}, R\right)$ by equation (2) one might be inclined to say that $z_{0}$ is an essential isolated singularity of $f$ if and only if the point $z_{0}$ is an essential isolated singularity of at least one of the functions $f_{k}$ for some $k \geqq-n$ in analogy with the definition of an essential isolated singularity for polyanalytic functions. We will see later that the above definition of an essential isolated singularity for a multianalytic function is unduly restrictive. For the purposes of this paper the following somewhat more general definition of essential isolated singularity for a multianalytic function turns out to be appropriate. To this end let $f$ be multianalytic at $z_{0}$ and suppose that $f$ is represented on $A\left(z_{0}, R\right)$ by equation (2). Later we shall show that the functions $f_{k}$ in equation (2) are uniquely determined on $A\left(z_{0}, R\right)$ by $f$. Consequently the following definitions are not ambiguous. For each $k \geqq-n$, we now define $d_{k}=d\left(f_{k}\right)$ the order of $f_{k}$ at $z_{0}$ as follows. If $f_{k} \equiv 0$ on $A\left(z_{0}, R\right)$, then $d_{k}=-\infty$. If $z_{0}$ is an essential isolated singularity, then $d_{k}=+\infty$. Now suppose that $f_{k} \not \equiv 0$ on $A\left(z_{0}, R\right)$ and that $z_{0}$ is not an essential isolated singularity of $f_{k}$. If $z_{0}=\infty$, then there is some unique integer $\nu$ so that $f_{k}(z) / z^{\nu}$ is analytic and not zero at $z_{0}=\infty$. In this case we set $d_{k}=\nu$. If $z_{0} \neq \infty$, then there is some unique integer $\nu$ so that $\left(z-z_{0}\right)^{\nu} f_{l}(z)$ is analytic and not zero at $z_{0}$. In this case we set $d_{k}=\nu$. Note that $d_{k}$ is either an integer or $\pm \infty$. We now define $d=d(f)$ the order of $f$ at $z_{0}$ to be the least upper bound of the numbers $d_{k}-k$ for $k \geqq-n$. Note that $d$ is either an integer or $\pm \infty$. Observe also that $d=-\infty$ if and only if $f \equiv 0$ on $A\left(z_{0}, R\right)$. We now say that $z_{0}$ is an essential isolated singularity of $f$ if and only if $d=+\infty$. We also say that $z_{0}$ is a nonessential isolated singularity of $f$ or a pole of $f$ of order $d$ if and only if $d \neq+\infty$.

Note in particular that if the point $z_{0}$ is an essential isolated singularity of at least one of the functions $f_{k}$ for some $k \geqq-n$, then according to the above definition the point $z_{0}$ is an essential isolated singularity of $f$. Note further that $z_{0}$ can be an essential isolated singularity of $f$ even though the point $z_{0}$ is not an essential isolated singularity of any of the $f_{k}$.

Now let $f$ be a function which is multianalytic at a point $z_{0}$. If $\alpha$ is any finite complex number, then $\alpha$ is termed an exceptional value for $f$ at $z_{0}$ if and only if there is some $R>0$ so that $f-\alpha$ is defined and never zero on $A\left(z_{0}, R\right)$. We wish to introduce a somewhat more general notion of an exceptional value for $f$ at $z_{0}$. Suppose that $g$ is any function which is multianalytic at $z_{0}$ and which is such that $z_{0}$ is not an essential isolated singularity of $g$. Then $g$ is termed an exceptional value for $f$ at $z_{0}$ if and only if there is some $R>0$ so that $f-g$ is defined and is never zero on $A\left(z_{0}, R\right)$. If $g$ and $h$ are two exceptional values for $f$ at $z_{0}$, we say that these exceptional 
values $g$ and $h$ are distinct if and only if there is some $R>0$ so that $g-h$ is defined and not identically zero on $A\left(z_{0}, R\right)$.

With the aid of the above terminology we can now state our main result in the following concise form.

THEOREM. Let $f$ be a function which is multianalytic at a point $z_{0}$, finite or infinite. If $z_{0}$ is an essential isolated singularity of $f$, then $f$ can have at most one exceptional value at $z_{0}$.

First note that the above theorem is sharp in the sense that if $z_{0}$ is not an essential isolated singularity of $f$, then $f$ has at least two exceptional values. In fact if $\alpha$ in any nonzero finite complex number, then $f-\alpha$ is clearly an exceptional value for $f$ at $z_{0}$.

Before proceeding to a demonstration of the above theorem one observation seems to be in order. It is natural to ask if the above theorem remains valid if we employ a somewhat more general definition of multianalytic function. One might be tempted to say that a function $f$ is multianalytic in $A\left(z_{0}, R\right)$ if and only if there exists a sequence of functions $\left\{f_{k}\right\}$ analytic in $A\left(z_{0}, R\right)$ for $k=0, \pm 1, \pm 2, \cdots$, such that

$$
\left.f(z)=\sum_{k=-\infty}^{+\infty} \overline{\left(z-z_{0}\right.}\right)^{k} f_{k}(z),
$$

for all $z$ in $A\left(z_{0}, R\right)$ where suitable assumptions are made in regard to the convergence of the above series. In this series it is understood that if $z_{0}=\infty$, then the expression $\overline{\left(z-z_{0}\right)^{k}}$ is replaced by $1 / \bar{z}^{k}$ for $k=0, \pm 1, \pm 2, \cdots$. If such a definition of multianalytic function is adopted then the above theorem becomes false. The difficulty of course

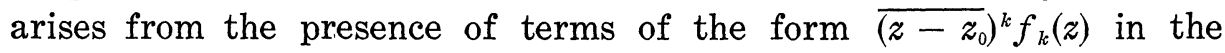
above series for infinitely many $k \leqq 0$. As a particularly simple example we take $f(z)=e^{z} e^{\bar{z}}$. Observe that

$$
f(z)=\sum_{k=0}^{+\infty} \bar{z}^{k} \frac{e^{z}}{k !}
$$

for all $z$. This function $f$ is certainly multianalytic at the point $z_{0}=\infty$ according to the provisional definition of this paragraph. It is also reasonable to regard the point $z_{0}=\infty$ as an essential isolated singularity of this function $f$. This function $f$, however, assumes only positive real values.

Observe that if $f$ is multianalytic and of order $d$ at a finite point $z_{0}$, then $g(z)=f\left(z+z_{0}\right)$ is multianalytic and of the same order $d$ at the point 0 . Also if $f$ is multianalytic and is of order $d$ at the point 0 , then $g(z)=f(1 / z)$ is multianalytic and of the same order $d$ at the point $\infty$. From the above observations we see that in order to establish the above theorem that there is no loss in generality in assuming that 
the essential isolated singularity is at the point $z_{0}=\infty$.

In what follows we shall invariable assume that $f$ is a given function which is multianalytic at the point $z_{0}=\infty$. Consequently there is some $R>0$ and some nonnegative integer $n \geqq 0$ and a sequence $\left\{f_{k}\right\}$ of functions analytic on $A(\infty, R)$ for all $k \geqq-n$ such that

$$
f(z)=\sum_{k=-n}^{+\infty} \frac{f_{k}(z)}{\bar{z}^{k}}
$$

for all $z$ in $A(\infty, R)$, where $\bar{z}$ denotes the complex conjugate of $z$ and where it is understood that the series in equation (3) is almost uniformly convergent in $A(\infty, R)$. Also in what follows it will be convenient to assume that for each $k \geqq-n$, that the Laurent series expansion of $f_{k}$ with center $z_{0}=\infty$ is given by

$$
f_{k}(z)=\sum_{\mu=-\infty}^{+\infty} \alpha_{\mu}^{(k)} z^{\mu}
$$

for all $|\boldsymbol{z}|>R$.

We now need the following result.

Lemma 1. For $\rho>R$, the series

$$
\sum_{k=-n}^{+\infty} z^{k} \frac{f_{k}(z)}{\rho^{2 k}}
$$

is almost uniformly convergent in $R<|z| \leqq \rho$. Also the above series is almost uniformly absolutely convergent in $R<|z|<\rho$.

Proof. First observe that the general term of the series in equation (5) may be written in the form $z^{k} f_{k}(z) / \rho^{2 k}=\left(z \bar{z} / \rho^{2}\right)^{k} f_{k}(z) / \bar{z}^{k}$. Now the sequence $\left\{\left(z \bar{z} / \rho^{2}\right)^{k}\right\}$ is monotone decreasing and uniformly bounded for $R<|z| \leqq \rho$. Consequently by Abel's theorem the series in equation (5) is almost uniformly convergent in $R<|z| \leqq \rho$. Next let $R<\mu<$ $\lambda<\rho$. Since the series in equation (3) is uniformly convergent on $\mu \leqq$ $|z| \leqq \lambda$ we see that there is some positive constant $M$ so that $\left|f_{k}(z) / \bar{z}^{k}\right| \leqq$ $M$ for $\mu \leqq|z| \leqq \lambda$ and for $k \geqq-n$. Thus if $\mu \leqq|z| \leqq \lambda$ we see that $\left|z^{k} f_{k}(z) / \rho^{2 k}\right| \leqq(\lambda / \rho)^{2 k} M$ for all $k \geqq-n$. Consequently by the Weierstrass test, the series in equation (5) is uniformly absolutely convergent in $\mu \leqq|z| \leqq \lambda$. Thus the series in equation (5) is almost uniformly absolutely convergent in $R<|z|<\rho$. This completes the proof of the lemma.

In view of the above lemma we may now give the following definition. For each $\rho>R$, it will be formed convenient to introduce the auxiliary function $f(z, \rho)$ defined by the condition that 


$$
f(z, \rho)=\sum_{k=-n}^{+\infty} z^{k} \frac{f_{k}(z)}{\rho^{2 k}},
$$

for all $z$ in $R<|z| \leqq \rho$. Note that the function $f(z, \rho)$ is analytic on $R<|z|<\rho$ and continuous on $R<|z| \leqq \rho$. Moreover, $f(z, \rho)=$ $f(z)$ for all $z$ on $|z|=\rho>R$.

In some considerations it would be convenient if the series in equation (6) for $f(z, \rho)$ were almost uniformly convergent in the larger annulus $R<|z|<+\infty$ for all $\rho>R$. This is not the case however. In order to construct an example we first consider a power series $\sum a_{k} z^{k}, k \geqq 0$, with radius of convergence one which is uniformly and absolutely convergent on the closed disc $|z| \leqq 1$. Let $f(z)=\sum a_{k} z^{k} / \bar{z}^{k}$, $k \geqq 0$, for all $|z|>0$. This function $f$ is multianalytic in $A(\infty, 0)$. If $\rho>0$, then $f(z, \rho)=\sum a_{k} z^{2 k} / \rho^{2 k}, k \geqq 0$, is absolutely and uniformly convergent in $0 \leqq|z| \leqq \rho$ and divergent elsewhere.

Next we have the following uniqueness result which we have appealed to earlier.

Lemma 2. Let the function $f$ be multianalytic on $A\left(z_{0}, R\right)$ and assume that $f$ is represented on $A\left(z_{0}, R\right)$ by equation (2). Then the functions $f_{k}$ in equation (2) are uniquely determined on $A\left(z_{0}, R\right)$ by the function $f$.

Proof. There is no loss in generality in assuming that $z_{0}=\infty$ and that $f$ is represented on $A(\infty, R)$ by equations (3) and (4). It now suffices to show that if $f \equiv 0$ on $A(\infty, R)$, then $f_{k} \equiv 0$ on $A(\infty, R)$ for all $k \geqq-n$. Since $f \equiv 0$ on $A(\infty, R)$, then $f(z, \rho)=$ 0 for all $|z|=\rho>R$. If $C_{\rho}=C(0, \rho)$, then from equation (6) we see that

$$
\sum_{k=-n}^{+\infty} \frac{a_{\nu-k}^{(k)}}{\rho^{2 k}}=\frac{1}{2 \pi i} \int_{C_{\rho}} \frac{f(z, \rho) d z}{z^{1+\nu}}=0,
$$

for all $\rho>R$ and for every integer $\nu$. Hence $a_{\nu-k}^{(k)}=0$ for all $k \geqq-n$ and for every integer $\nu$. Hence $a_{k^{\prime}}^{(k)}=0$ for all $k \geqq-n$ and for every integer $\mu$. Consequently $f_{k} \equiv 0$ on $A(\infty, R)$ for each $k \geqq-n$. This completes the proof of the lemma.

It is desirable at this state to give a rough indication of how we propose to establish our theorem. To this end let $f$ be multianalytic on $A(\infty, R)$ and let $S>R$. Now let $F$ denote the collection of all functions $f(\rho z, \rho)$ for which $\rho>S$. The functions belonging to $F$ are all analytic in the annulus $R / S<|z|<1$. We will now base a proof of our theorem on the establishment of a suitable connection between the behavior of $f$ on $A(\infty, R)$ and the behavior of the family $F$ on the annulus $R / S<|z|<1$. Our proof is analogous to the proof of 
Picard's big theorem for analytic functions which employs Montel's criterion for normal families of analytic functions.

It is with the above point of view that we offer the following lemmas.

Lemma 3. Let $f$ be multianalytic and not identically zero on $A(\infty, R)$ and suppose that $f$ has a pole of order $d$ at the point $\infty$. Then there exists an isolated set $Z$ of $A(0,1)$ with the following property. For any nonempty closed subset $B$ of $A(0,1)$ which does not meet $Z$, there exist positive constants $L, K$, and $\rho_{0}$, which in general depend on the set $B$, so that $L \rho^{d}<|f(\rho z, \rho)|<K \rho^{d}$ for all $z$ in $B$ and for all $\rho>\rho_{0}$.

Proof. It suffices to find a function $P(z)$ which is analytic and not identically zero in $A(0,1)$ such that $f(\rho z, \rho) / \rho^{d} \rightarrow P(z)$ as $\rho \rightarrow+\infty$ almost uniformly with respect to $z$ in $A(0,1)$. We may then take $Z$ to denote the set of zeros of $P(z)$ in $A(0,1)$. With the above goal in mind we proceed as follows. Let $f$ be represented on $A(\infty, R)$ by equations (3) and (4). Let $\sigma>R$ be fixed. From the almost uniform convergence of the series in equation (3) we see that there is some positive constant $M$ so that $\left|f_{k}(z) / \bar{z}^{k}\right| \leqq M$ for $|z|=\sigma$ for all $k \geqq-n$. Hence $\left|f_{k}(z) / z^{d_{k}+1}\right| \leqq M / \sigma^{d_{k}-k+1}$ for $|z|=\rho$ and $k \geqq-n$. Now for each $k \geqq-n$, the functions $f_{k}(z) / z^{d_{k}+1}$ are analytic on $N(\infty, R)$ and have a zero of order one at the point $z_{0}=\infty$. Hence from Schwarz's lemma we deduce the estimate

$$
\left|f_{k}(z)\right| \leqq \frac{|z|^{d_{k}} M}{\sigma^{d_{k}-k}}
$$

which is valid for $|z| \geqq \sigma$ and for all $k \geqq-n$. We shall appeal to the above estimate several times in the course of our argument. Now for each $k \geqq-n$, let $a_{k}=a_{\nu}^{(k)}$ where $\nu=d_{k}$. Next for each $k \geqq-n$, let $b_{k}=a_{k}$ if $d_{k}-k=d$ and let $b_{k}=0$ if $d_{k}-k \leqq d-1$. Also for each $k \geqq-n$, let $c_{k}=a_{k}-b_{k}$. Observe that $b_{k} \neq 0$ for at least one $k \geqq-n$. If we now integrate $f_{k}(z) / z^{d_{k}+1}$ around the circumference $C(0, \sigma)$ then from inequality (7) we deduce the estimate

$$
\left|a_{k}\right| \leqq \frac{M}{\sigma^{d_{k}-k}},
$$

which is valid for all $k \geqq-n$. From the above inequality, it follows that the series $\sum b_{k} z^{2 k}, k \geqq-n$, is convergent in the annulus $A(0,1)$. Now let

$$
P(z)=z^{d} \sum_{k=-n}^{+\infty} b_{k} z^{2 k}
$$


for $z$ in $A(0,1)$. Evidently $P(z)$ is analytic and not identically zero in the annulus $A(0,1)$. Next if $k \geqq-n$ and if $\rho|z|>\sigma$ we see from inequality (8) that $\left|a_{k} \rho^{d_{k}-k} z^{d_{k}+k}\right| \leqq|\rho z / \sigma|^{d} M$. Hence for each $\rho>\sigma$, the series $\sum a_{k} \rho^{d_{k}-k} z^{d_{k}+k}, k \geqq-n$, is convergent for $z$ in $\sigma / \rho \leqq|z|<1$. Next for each $k \geqq-n$, we see from inequalities (7) and (8) that $\left|f_{k}(z) / z^{d_{k}}-a_{k}\right| \leqq 2 M / \sigma^{d_{k}-k}$ for $|z|=\sigma$. Now for each $k \geqq-n$, the function $f_{k}(z) / z^{d_{k}}-a_{k}$ is analytic in $N(\infty, R)$ and has a zero of order at least one at the point $z_{0}=\infty$. Hence by Schwarz's lemma we see that $\left|f_{k}(z) / z^{d_{k}}-a_{k}\right| \leqq 2 M /|z| \sigma^{d_{k}-k-1}$ for $k \geqq-n$ and $|z|>\sigma$. Consequently we have that $\left|z^{k} f_{k}(\rho z) / \rho^{k}-a_{k} \rho^{d_{k}-k} z^{d_{k}+k}\right| \leqq 2|z|^{2 k}|\rho z / \sigma|^{d-1} M$ for $k \geqq-n$ and $\rho|z| \geqq \sigma$. Hence it follows that

$$
\left|f(\rho z, \rho)-\sum_{k=-n}^{+\infty} a_{k} \rho^{d_{k}-k} z^{d_{k}+k}\right| \leqq \frac{2 M|z|^{-2 n}}{1-|z|^{2}}\left|\frac{\rho z}{\sigma}\right|^{d-1},
$$

for $\rho>\sigma$ and $\sigma / \rho \leqq|z|<1$. Next from inequality (8) observe that $\left|c_{k} \rho^{d_{k}-k} z^{d_{k}+k}\right| \leqq|z|^{2^{k}} M|\rho z / \sigma|^{d-1}$ for $\rho|z| \geqq \sigma$ and $k \geqq-n$. Hence the series $\sum c_{k} \rho^{d_{k}-k} z^{d_{k}+k}, k \geqq-n$, is convergent for $\rho>\sigma$ and $\sigma / \rho \leqq|z|<1$. In addition we have the estimate

$$
\left|\sum_{k=-n}^{+\infty} c_{k} \rho^{d_{k}-k} z^{d_{k}+k}\right| \leqq \frac{M|z|^{-2 n}}{1-|z|^{2}}\left|\frac{\rho z}{\sigma}\right|^{d-1},
$$

which is valid for $\rho>\sigma$ and $\sigma / \rho \leqq|z|<1$. Now from equation (9) and inequalities (10) and (11) we deduce that

$$
\begin{aligned}
& \left|f(\rho z, \rho)-\rho^{d} P(z)\right|=\left|f(\rho z, \rho)-\sum_{k=-n}^{+\infty} b_{k} \rho^{d_{k}-k} z^{d_{k}+k}\right| \\
\leqq & \left|f(\rho z, \rho)-\sum_{k=-n}^{+\infty} a_{k} \rho^{d_{k}-k} z^{d_{k}+k}\right|+\left|\sum_{k=-n}^{+\infty} c_{k} \rho^{d_{k}-k} z^{d_{k}+k}\right| \\
\leqq & \left.\left|\frac{3 M|z|^{-2^{n}}}{1-|z|^{2}}\right| \frac{\rho z}{\sigma}\right|^{d-1},
\end{aligned}
$$

for $\rho>\sigma$ and $\sigma / \rho \leqq|z|<1$. Hence from the above inequality we finally obtain the estimate

$$
\left|\frac{f(\rho z, \rho)}{\rho^{d}}-P(z)\right| \leqq \frac{1}{\rho} \frac{3 M|z|^{-2 n}}{1-|z|^{2}}\left|\frac{z}{\sigma}\right|^{d-1},
$$

which is valid for $\rho>\sigma$ and $\sigma / \rho \leqq|z|<1$. The result now follows from the above estimate.

The above lemma has the following partial converse.

LeMma 3. Let $f$ be multianalytic on $A(\infty, R)$ and let $0<\lambda \leqq 1$ be fixed and suppose that $\left\{\rho_{m}\right\}$ is a sequence of real numbers greater than $R$ which diverges to $+\infty$. Suppose that there is some positive constant $K$ and some integer $t$ so that 


$$
|f(\rho z, \rho)| \leqq K \rho^{t},
$$

for $|z|=\lambda$ and $\rho=\rho_{m}$. Then $z_{0}=\infty$ is a pole of $f$ of order $d \leqq t$.

Proof. Assume that $f$ is represented on $A(\infty, R)$ by equations (3) and (4). If $C_{\lambda}=C(0, \lambda)$, then

$$
\sum_{k=-n}^{+\infty} a_{\nu-k}^{(k)} \rho^{\nu-2 k}=\frac{1}{2 \pi i} \int_{C_{\lambda}} \frac{f(\rho z, \rho) d z}{z^{1+\nu}},
$$

for $\rho>R$ and for any integer $\nu$. Consequently

$$
\left|\sum_{k=-n}^{+\infty} \frac{a_{\nu-k}^{(k)}}{\rho^{2 k+t-\nu}}\right| \leqq \frac{K}{\lambda^{\nu}},
$$

for $\rho=\rho_{m}$ and for any integer $\nu$. Now in the above infinite sum it is easy to see that the coefficients of positive powers of $\rho$ must vanish. Hence $a_{\nu \rightarrow k}^{(k)}=0$ for $k \geqq-n$ and $2 k+t-\nu \leqq-1$. Hence $a_{\mu}^{(k)}=0$ for $k \geqq-n$ and $\mu \geqq k+t+1$. Hence $z_{0}=\infty$ is a removable singularity or a pole of $f_{k}$ of order $d_{k} \leqq k+t$ for all $k \geqq-n$. Hence the point $z_{0}=\infty$ is a pole of $f$ of order $d \leqq t$ as claimed.

The above result is somewhat technical in nature. The following special case of Lemma 3 is perhaps more revealing as to the basic content of Lemma 3 .

COROLlary 1. Let $f$ be multianalytic on $A(\infty, R)$ and let $\left\{\rho_{m}\right\}$ be a sequence of real numbers greater than $R$ diverging to $+\infty$. Suppose that there is some positive constant $K$ and some integer $t$ so that

$$
|f(z)| \leqq K|z|^{t},
$$

for $|z|=\rho_{m}$. Then $z_{0}=\infty$ is a pole of $f$ of order $d \leqq t$.

As a special case of Corollary 1 we have the following result which is a generalization of Liouville's theorem.

Corollary 2. A polyentire function which is bounded above in the finite complex plane is constant function.

It is now natural to ask if Lemma 3 is still valid if the inequality (12) is reversed. If this were the case, then Corollary 1 would also be valid when the inequality (13) is reversed. This leads to the following question. Suppose that $f$ is multianalytic in $A(\infty, R)$ and that $\left\{\rho_{m}\right\}$ is a sequence of real numbers greater than $R$ diverging to $+\infty$ and that $K$ is some positive constant such that $|f(z)|>K$ for $|z|=\rho_{m}$. Does it then necessarily follow that the point $z_{0}=\infty$ is not an essential isolated singularity of $f$ ? The answer is no. As an 
example consider the function $f(z)=\exp (1 / z \bar{z}) \sin z$. Evidently

$$
f(z)=\sum_{k=0}^{+\infty} \frac{\sin z}{k ! z^{k} \bar{z}^{k}},
$$

for all $|z|>0$, the convergence being almost uniform in $0<|z|<+\infty$. This function $f$ is clearly multianalytic in $A(\infty, 0)$ and has the point $z_{0}=\infty$ as an essential isolated singularity. Now let $\rho_{m}=m \pi+\pi / 2$ for $m=0,1,2, \cdots$. It is easy to see that there is some positive constant $K$ so that $|f(z)| \geqq|\sin z| \geqq K$ for $|z|=\rho_{m}$. However, it is possible to establish a useful variant of Lemma 3 when the inequality (12) is reversed if we impose some suitable additional restrictions. Our next few lemmas lead up to such a result.

Assume again that $f$ is multianalytic in $A(\infty, R)$ and is given by equations (3) and (4). For each $k \geqq-n$, let

$$
\left\{\begin{array}{l}
g_{k}(z)=\sum_{\mu=-k}^{+\infty} a_{\mu}^{(k)} z^{\mu}, 0<|z|<+\infty, \\
h_{k}(z)=\sum_{\mu=-\infty}^{-k-1} a_{\mu}^{(k)} z^{\mu}, 0<|z|<+\infty .
\end{array}\right.
$$

We now have the following result.

Lemma 4. The two series

$$
\sum_{k=-n}^{+\infty} \frac{g_{k}(z)}{\bar{z}^{k}}, \sum_{k=-n}^{+\infty} \frac{h_{k}(z)}{k=-n}
$$

are almost uniformly convergent in $R<|z|<+\infty$.

Proof. Let $\sigma>R$ be fixed. The series in equation (3) is uniformly convergent on $|z|=\sigma$. Hence there is some positive constant $M$ so that $\left|f_{k}(z) / \bar{z}^{k}\right| \leqq M$ for $|z|=\sigma$ and $k \geqq-n$. If $C_{\sigma}=C(0, \sigma)$ and if $|z|>\sigma$, then it is easy to see that

$$
\frac{h_{k}(z)}{\bar{z}^{k}}=-\frac{1}{2 \pi i} \int_{C_{\sigma}} \frac{\sigma^{2 k}}{|z|^{2 k}} \frac{f_{k}(\zeta)}{\bar{\zeta}^{k}} \frac{d \zeta}{\zeta-z},
$$

for each $k \geqq-n$. From the above equation we see that $\left|h_{k}(z) / \bar{z}^{k}\right| \leqq$ $(\sigma /|z|)^{2 k} M \sigma /(|z|-\sigma)$, for $|z|>\sigma$ and $k \geqq-n$. From this last inequality it follows that the second series in (15) is almost uniformly absolutely convergent in $R<|z|<+\infty$. From the identity

$$
\sum_{k=-n}^{m} \frac{g_{k}(z)}{\bar{z}^{k}}=\sum_{k=-n}^{m} \frac{f_{k}(z)}{\bar{z}^{k}}-\sum_{k=-n}^{m} \frac{h_{k}(z)}{\bar{z}^{k}},
$$

which is valid for $m \geqq-n$ and $|z|>R$, we see that the first series in (15) is almost uniformly convergent in $R<|z|<+\infty$. This com- 
pletes the proof of the lemma.

In view of the above lemma we can now make the following definition. Let $f$ be multianalytic on $A(\infty, R)$ and assume that $f$ is given by equations (3) and (4). For each $k \geqq-n$, let $g_{k}$ and $h_{k}$ be given by equation (14). We then define the functions $g$ and $h$ by the condition that

$$
\left\{\begin{array}{l}
g(z)=\sum_{k=-n}^{+\infty} \frac{g_{k}(z)}{\bar{z}^{k}}, \\
h(z)=\sum_{k=-n}^{+\infty} \frac{h_{k}(z)}{\bar{z}^{k}},
\end{array}\right.
$$

for all $z$ is $A(\infty, R)$. From Lemma 4, the convergence of the series in equation (16) is almost uniform on $A(\infty, R)$. Consequently the functions $g$ and $h$ are multianalytic on $A(\infty, R)$. Note also that $f=$ $g+h$. Observe that $z_{0}=\infty$ is a pole of $h$ of order $d \leqq 2 n-1$. Hence $z_{0}=\infty$ is an essential singularity of $f$ if and only if $z_{0}=\infty$ is an essential singularity of $g$. Note also that $f(z, \rho)=g(z, \rho)+h(z, \rho)$ for $R<|z| \leqq \rho$. In the considerations that follow, the function $h(z, \rho)$ will play the role of a negligible error term so that certain properties of the function $f(z, \rho)$ will be inherited by the function $g(z, \rho)$. The function $g(z, \rho)$ is easier to deal with, however, than the function $f(z, \rho)$ because of the following result.

Lemma 5. For $\rho>R$, the auxiliary function $g(z, \rho)$ can be extended to $0 \leqq|z| \leqq \rho$ in such a way that $g(z, \rho)$ is continuous on $0 \leqq|z| \leqq \rho$ and analytic on $0 \leqq|z|<\rho$. Moreover, this unique extension is given by

$$
g(z, \rho)=\sum_{k=-n}^{+\infty} z^{k} \frac{g_{k}(z)}{\rho^{2 k}},
$$

for $0 \leqq|z| \leqq \rho$, the series in equation (17) being uniformly convergent on $0 \leqq|z| \leqq \rho$ and almost uniformly absolutely convegent on $0 \leqq|z|<\rho$.

Proof. Let $\rho>R$ be fixed. Then from Lemma 1, we see that the series in equation (17) is uniformly convergent on $|\boldsymbol{z}|=\rho$. However, for each $k \geqq-n$, we see from equation (14) that the functions $z^{k} g_{k}(z)$ are actually entire functions. Consequently from the maximum modulus principle we see that the series in equation (17) is uniformly convergent on $0 \leqq|z| \leqq \rho$. It is easy to see that the series in equation (17) is almost uniformly absolutely convergent in $0 \leqq|z|<\rho$. This completes the proof of the lemma.

Now assume for the moment that the function $g$ is not identically zero in $A(\infty, R)$. This will be the case for example when the function 
$f$ has an essential singularity at the point $z_{0}=\infty$. It is not difficult to see that there is some $R_{0}>R$ so that if $\rho>R_{0}$, then $g(z, \rho)$ is not identically zero in $0 \leqq|z| \leqq \rho$. For $\rho>R_{0}$, let $s \geqq 0$ denote the multiplicity of the root $z=0$ of $g(z, \rho)$ and let $a_{1}, a_{2}, \cdots, a_{q}$ be the $q \geqq 0$ nonzero roots of $\mathrm{g}(z, \rho)$ in $0<|z|<\rho$ with due regard for multiple roots. Finally for $\rho>R_{0}$, let the power series expansion of $g(z, \rho)$ with center $z_{0}=0$ and radius at least $\rho$ be given by

$$
g(z, \rho)=c_{s} z^{s}+c_{s+1} z^{s+1}+\cdots,
$$

for $0 \leqq|z|<\rho$. Of course $s \geqq 0$ and $c_{s} \neq 0$. Now $s, q$, and $a_{1}, a_{2}, \cdots$, $a_{q}$, and $c_{s}$ depend in general upon $\rho$ for $\rho>R_{0}$. The following result gives some information concerning this dependence.

Lemma 6. Assume that the function g given in equation (16) is not identically zero on $A(\infty, R)$. Then there is some $R_{0}>R$ and there is some nonnegative integer $m$ and some positive constants $K_{1}$ and $K_{2}$ such that the following is true. If $\rho>R_{0}$, then $g(z, \rho)$ is not identically zero in $0 \leqq|z| \leqq \rho$, and $s$ has a constant value independent of $\rho$, and

$$
\left|c_{s}\right|<K_{1} \rho^{2(n-m)}
$$

and

$$
\left|a_{1}\right|,\left|a_{2}\right|, \cdots,\left|a_{q}\right|>\frac{K_{2}}{\rho^{2 m}}
$$

Proof. If we consider the series expansion for $g(z, \rho)$ in equation (17) which is valid for $\rho>R$ and $0 \leqq|z| \leqq \rho$, it is not difficult to verify the following. There exist integers $s \geqq 0$ and $m \geqq 0$ and there exist entire functions $l_{k}$ for $k \geqq 0$ such that $l_{0}(0)=0, l_{1}(0)=0, \cdots, l_{m-1}(0)=0$, but $l_{m}(0) \neq 0$, and such that

$$
\begin{aligned}
& g(z, \rho)=z^{s} \rho^{2 n} l(z, \rho), \\
& l(z, \rho)=z \sum_{k=0}^{m-1} \frac{l_{k}(z)}{\rho^{2 k}}+\sum_{k=m}^{+\infty} \frac{l_{k}(z)}{\rho^{2 k}},
\end{aligned}
$$

for $\rho>R$ and $0 \leqq|z| \leqq \rho$. Now there is some $R_{1}>R$ so that $l(0$, $\rho) \neq 0$ for $\rho>R_{1}$. Hence if $\rho>R_{1}$, then $g(z, \rho)$ is not identically zero in $0 \leqq|z| \leqq \rho$ and $z=0$ is a root of $g(z, \rho)$ of exact multiplicity s. Next $c_{s}=\rho^{2 n} l(0, \rho)$ for $\rho>R_{1}$. Hence there is some positive constant $K_{1}$ and some $R_{2}>R_{1}$ so that $\left|c_{s}\right|<K_{1} \rho^{2(n-m)}$ for all $\rho>R_{2}$. Next let $0<\sigma<R_{2}$ be fixed. Then there are positive constants $K$ and $L$ and some $R_{3}>R_{2}$ so that 


$$
|l(z, \rho)| \geqq \frac{L}{\rho^{2 m}}-|z| K,
$$

for $0 \leqq|z| \leqq \sigma$ and $\rho>R_{3}$. If we set $K_{2}=L / K$ we see that $|l(z, \rho)|>0$ whenever $\rho>R_{3}$ and $0 \leqq|z| \leqq \sigma$ and $|z|<K_{2} / \rho^{2 m}$. Now choose $R_{0}>$ $R_{3}$ so that $K_{2} / \rho^{2 m}<\sigma$ whenever $\rho>R_{0}$. Thus if $\rho>R_{0}$ and if $0 \leqq$ $|z| \leqq K_{2} / \rho^{2 m}$, then $|l(z, \rho)|>0$. Consequently $g(z, \rho) \neq 0$ for $\rho>R_{0}$ and $0<|\boldsymbol{z}| \leqq K_{2} / \rho^{2 m}$. Inequality (19) now follows from this last estimate. This completes the proof of the lemma.

Next for purposes of completeness we would like to mention some basic definitions and some elementary results from the Nevanlinna theory of meromorphic functions [5]. First for $x \geqq 0$ we define the so called $\log$ plus function $\log ^{+} x$ defined by the condition that $\log ^{+} x=0$ if $0 \leqq x<1$ and $\log ^{+} x=\log x$ if $x \geqq 1$. Now let $R>0$ and suppose that $f$ is a function which is nonconstant and meromorphic in $0 \leqq$ $|z|<R$. For $0<r<R$ we define the expression $m(r, f)$ by the condition that

$$
m(r, f)=\frac{1}{2 \pi} \int_{0}^{2 \pi} \log ^{+}\left|f\left(r e^{i \phi}\right)\right| d \phi .
$$

Next for $0<r<R$ we define the expression $N(r, f)$ by the condition that

$$
N(r, f)=\sum_{i=1}^{q} \log \frac{r}{\left|b_{i}\right|},
$$

where $b_{1}, b_{2}, \cdots, b_{q}$ are the $q \geqq 0$ nonzero poles of $f$ in $0<|\boldsymbol{z}|<r$ with due regard for multiplicities. Also for $0<r<R$ we define the expression $T(r, f)$ by the condition that

$$
T(r, f)=m(r, f)+N(r, f) .
$$

The function $T(r, f)$ is called the characteristic function of $f$ and plays a fundamental role in the theory of meromorphic functions. We now mention the following result. Suppose that $f$ has a zero of order $s$ or a pole of order $-s$ at $z=0$ so that the Laurent series expansion of $f$ with center $z_{0}=0$ is

$$
f(z)=c_{s} z^{s}+c_{s+1} z^{s+1}+\cdots,
$$

where $c_{s} \neq 0$. We then have that

$$
T(r, f)=T\left(r, \frac{1}{f}\right)+\log \left|c_{s}\right| r^{s},
$$

for $0<r<R$ [5, p. 4]. We need also to cite one other result. Suppose that $f$ is analytic and nonconstant in $0 \leqq|z|<R$. If $0<$ 
$r<R$ and if $0<\lambda<1$, then

$$
\log ^{+} M(\lambda r, f) \leqq \frac{1+\lambda}{1-\lambda} T(r, f)
$$

where $M(\lambda r, f)$ denotes the maximum modulus of $f$ on $|z|=\lambda r \quad$ [5, p. 18].

We are now ready to state and prove an analogue of Lemma 3 which we have alluded to earlier. For this purpose it is convenient to introduce the following definition. Let $f$ be anbitrary complex valued function which is defined and continuous on $A(\infty, R)$. If $\rho>$ $R$ and if $f$ does not vanish on the circumference $C(0, \rho)$, we define the expression $\Delta_{\rho} f$ to be $1 / 2 \pi$ times the change in the argument of $f$ on the positively oriented circumference $C(0, \rho)$. We now have the following result.

Lemma 7. Let $f$ be multianalytic on $A(\infty, R)$ and let $0<\lambda \leqq 1$ be fixed and suppose that there is some sequence $\left\{\rho_{m}\right\}$ of real numbers greater than $R / \lambda$ diverging to $+\infty$ and some positive constant $K$ and some integer $t$ so that

$$
|f(\rho z, \rho)| \geqq K \rho^{t},
$$

for $|\boldsymbol{z}|=\lambda$ and $\rho=\rho_{m}$. Suppose further that the sequence of integers $\Delta_{\lambda} f(\rho z, \rho)$ for $\rho=\rho_{m}$ is bounded above. Then the point $z_{0}=\infty$ is not an essential isolated singularity of $f$.

Proof. Suppose that $f$ is given on $A(\infty, R)$ by equation (3) and (4). First there is no loss in generality in assuming that the lower limit $n$ on summation in equation (3) is zero. Also there is no loss in generality in assuming that the exponent $t$ in inequality (25) is zero. For if the above two conditions are not met we need only consider the function $z^{n-t} f(z) / \lambda^{2 n-t} \bar{z}^{n}$. Next suppose that the functions $g$ and $h$ are given by equation (16). In equation (16) it is also understood that $n=0$. Let $\sigma>R$ be fixed. Since the series in equation (16) are uniformly convergent on $|\boldsymbol{z}|=\sigma$, there is some positive constant $M$ so that $\left|h_{k}(z) / \bar{z}^{k}\right| \leqq M$ for $|z|=\sigma$ and $k \geqq 0$. Hence $\left|z^{k} h_{k}(z)\right| \leqq M \sigma^{2 k}$ for $|z|=\sigma$ and $k \geqq 0$. Now for each $k \geqq 0$, the function $z^{k} h_{k}(z)$ is analytic in $N(\infty, R)$ and has zero at $z_{0}=\infty$ of at least order one. Hence by Schwarz's lemma we see that $\left|z^{k} h_{k}(z)\right| \leqq M \sigma^{2 k+1} /|z|$ for $|z| \geqq \sigma$ and $k \geqq 0$. Consequently $\left.\mid z^{k} h_{k}(\rho z) / \rho^{k}\right) \mid \leqq(\sigma M / \lambda \rho)(\sigma / \rho)^{2 k}$, for $|z|=\lambda$ and $\rho \geqq \sigma / \lambda$ and $k \geqq 0$. Hence $|h(\rho z, \rho)| \leqq(\sigma M / \lambda \rho) /\left(1-\sigma^{2} / \rho^{2}\right)$ for $|z|=\lambda$ and $\rho>\sigma / \lambda$. From the above inequality we see that $f(\rho z, \rho)-g(\rho z, \rho)=h(\rho z, \rho) \rightarrow 0$ as $\rho \rightarrow+\infty$ uniformly with respect to $z$ on the circumference $|z|=\lambda$. There is then some $R_{1}>R$ so 
that $|f(\rho z, \rho)-g(\rho z, \rho)|<K / 2$ for $|z|=\lambda$ and $\rho=\rho_{m}>R_{1}$. Hence $|g(\rho z, \rho)|>K / 2$ for $|z|=\lambda$ and $\rho=\rho_{m}>R_{1}$. Now $\mid f(\rho z, \rho)-g(\rho z$, $\rho)|<| f(\rho z, \rho) \mid$ for $|z|=\lambda$ and $\rho=\rho_{m}>R_{1}$. Hence $\Delta_{2} g(\rho z, \rho)=$ $\Delta_{2} f(\rho z, \rho)$ for $\rho=\rho_{m}>R_{1}$. Hence the sequence of integers $\Delta_{k} g(\rho z, \rho)$ for $\rho=\rho_{m}>R_{1}$ is bounded above. Let $p$ be some integer so that $\Delta_{i} g(\rho z, \rho) \leqq p$ for $\rho=\rho_{m}>R_{1}$. There is some $R_{0}>R_{1}$ so that the conclusions of Lemma 6 are in force for $g(z, \rho)$. It will also be convenient to assume that $R_{0}>1$. From inequation (19) and equation (21) we see that $N(\rho \lambda, 1 / g(z, \rho))<2 p(m+1) \log \rho-p \log K_{2}$ for $\rho=\rho_{m}>R_{0}$. From equation (20) we see that $m(\rho \lambda, 1 / g(z, \rho))<\log ^{+} 2 / K$ for $\rho=\rho_{m}>R_{0}$. From the last two estimates and from equation (22) it then follows that $T(\rho \lambda, 1 / g(z, \rho))<2 p(m+1) \log \rho-p \log K_{2}+\log ^{+} 2 / K$ for $\rho=$ $\rho_{m}>R_{0}$. If we remember that $n=0$ in inequality (18) we see that $\log \left|c_{s}(\rho \lambda)^{s}\right|<(s-2 m) \log \rho+s \log K_{1} \lambda$ for $\rho=\rho_{m}>R_{0}$. From the last two estimates and from equation (23) and from the fact that $R_{0}>1$ we see that there is some integer $q$ so that $T(\rho \lambda, g(z, \rho))<$ $q \log \rho$ for $\rho=\rho_{m}>R_{0}$. It will be convenient to assume that the integer $q$ is so chosen that $q>0$. Now from the above estimate and from inequality (24) we deduce that $\log ^{+} M(\rho \lambda / 2, g(z, \rho))<3 q \log \rho$ for $\rho=$ $\rho_{m}>R_{0}$. Since $R_{0}>1$ and $q>0$ it then follows that $|g(\rho z, \rho)|<\rho^{3 q}$ for $|z|=\lambda / 2$ and $\rho=\rho_{m}>R_{0}$. Hence by Lemma 3, the point $z_{0}=\infty$ is not an essential singularity of $g$. Thus the point $z_{0}=\infty$ is not an essential singularity of $f$. This completes the proof of the lemma.

From the foregoing lemma we now have the following corollary which is of some interest in itself.

Corollary 3. Let $f$ be multianalytic on $A(\infty, R)$. Suppose that $\left\{\rho_{m}\right\}$ is some sequence of real numbers greater than $R$ diverging to $+\infty$ and that $K$ is some positive constant so that

$$
|f(z)| \geqq K,
$$

for $|z|=\rho_{m}$. If $f$ does not vanish in $A(\infty, R)$, then the point $z_{0}=\infty$ is not an essential isolated singularity of $f$.

Note that the above result is sharp in the sense that the above result is false if we remove the condition that $f$ does not vanish in $A(\infty, R)$. As an example we may take the function $f(z)=\exp (1 / z \bar{z}) \sin z$ which we have considered earlier.

We are now finally in a proof of our theorem. The proof is now formally the same as the proof given in [4, p. 148]. Suppose now that $f$ is a function which is multianalytic on $A\left(z_{0}, R\right)$ and suppose further that the function $f$ admits two distinct exceptional values $g$ and $h$ at $z_{0}$. First there is no loss in generality in assuming that 
$z_{0}=\infty$. Also there is no loss in generality in assuming that $h \equiv 0$ on $A(\infty, R)$ and that $g$ is defined and $g \not \equiv 0$ on $A(\infty, R)$. Since $f$ and $f-g$ do not vanish on $A(\infty, R)$ there exist integers $r$ and $s$ such that $\Delta_{\rho} f=r$ and $\Delta_{\rho}(f-g)=s$ for all $\rho>R$. Consequently $\Delta_{\rho} f(z, \rho)=r$ and $\Delta_{\rho}(f(z, \rho)-g(z, \rho))=s$ for all $\rho>R$. Now there is some $\delta>\sigma>R$ and some integers $u$ and $v$ so that $\Delta_{\sigma} f(z, \rho)=u$ and $\Delta_{o}(f(z, \rho)-g(z, \rho))=v$ for all $\rho>\delta$. Hence if $\rho>\delta$, then the number of zeros of $f(z, \rho)$ in $\sigma<|z|<\rho$ is $(r-u)$ and the number of zeros of $f(z, \rho)-g(z, \rho)$ in $\sigma<|z|<\rho$ is $(s-v)$. Hence if $\rho>\delta$, then the number of zeros of $f(\rho z, \rho)$ in $\sigma / \rho<|z|<1$ is $(r-u)$ and the number of zeros of $f(\rho z, \rho)-g(\rho z, \rho)$ in $\sigma / \rho<$ $|z|<1$ is $(s-v)$. Now by Lemma 3 we can find $\sigma / \rho<a<b<1$ and some $\mu>\delta$ so that $L \rho^{t}<|g(\rho z, \rho)|<K \rho^{t}$ for $\mathrm{a}<|z|<b$ and $\rho>\mu$, where $L$ and $K$ are some positive constants and $t$ is some integer. Let $B$ denote the annulus $a<|z|<b$ and let $\left\{\rho_{m}\right\}$ be a sequence of real numbers greater than $\mu$ diverging to $+\infty$. For each $m \geqq 1$ define $H_{m}$ on $B$ by the condition that $H_{m}(z)=f\left(\rho_{m} z, \rho_{m}\right) / g\left(\rho_{m} z\right.$, $\rho_{m}$ ) for all $z$ in $B$. Let $H$ denote the set of these functions $H_{m}$ for all $m \geqq 1$. First observe that each function $H_{m}$ is analytic on $B$. Next observe that each function $H_{m}$ assumes the values zero and one at most $(r-u)$ and $(s-v)$ times, respectively, in $B$. Consequently the family $H$ is quasi-normal of order $q$ on $B$ where $q \leqq \min (r-u$, $s-v),[6, \mathrm{p} .67]$. There are now two cases to consider according as $q=0$ or $q \geqq 1$. Consider first the case when $q \geqq 1$. Then there exist $q$ distinct points $z_{1}, z_{2}, \cdots, z_{q}$ in $B$ and there exists a subsequence $H_{m_{v}}$ of $H_{m}$ such that $H_{m_{v}} \rightarrow \infty$ as $v \rightarrow+\infty$ almost uniformly on $B-$ $\left\{z_{1}, z_{2}, \cdots, z_{q}\right\}$. Hence there is some $a<\lambda<b$ and some $v_{0}$ so that $\left|H_{m_{v}}(z)\right| \geqq 1$ for $|z|=\lambda$ and $v \geqq v_{0}$. Hence $|f(\rho z, \rho)| \geqq|g(\rho z, \rho)| \rho>L_{\rho}^{t}$ for $|z|=\lambda$ and $\rho=\rho_{m_{v}}$ and $v \geqq v_{0}$. Also $\Delta_{\lambda} f(\rho z, \rho) \leqq \Delta_{\rho} f(z, \rho)=r$ for $\rho=\rho_{m_{v}}$ and $v \geqq v_{0}$. Hence by Lemma 7, the point $z_{0}=\infty$ is not an essential singularity of $f$. This establishes the theorem in the case that $q \geqq 1$. Consider next the case when $q=0$. Consequently the family $H$ is normal in $B$. There are now two possibilities to consider. First there may be a subsequence $H_{m_{v}}$ of $H_{m}$ such that $H_{m_{v}} \rightarrow \infty$ as $v \rightarrow+\infty$ almost uniformly on $B$. In this eventuality one shows as before that the point $z_{0}=\infty$ is not an essential singularity of $f$. Secondly there may be a subsequence $H_{m_{v}}$ of $H_{m}$ which converges almost uniformly on $B$. Choose $a<\lambda<b$. There is some constant $M$ so that $\left|H_{m_{v}}(z)\right| \leqq M$ for $|z|=\lambda$ and $v \geqq 0$. Hence $|f(\rho z, \rho)| \leqq$ $M|g(\rho z, \rho)|<M K_{\rho}^{t}$ for $|z|=\lambda$ and $\rho=\rho_{m_{v}}$ and $v \geqq 0$. Hence by Lemma 3 , the point $z_{0}=\infty$ is not an essential singularity of $f$. This establishes the theorem in the case that $q=0$. Thus in all cases we see that $z_{0}=\infty$ is not an essential singularity of $f$. This completes the proof of our theorem. 
As an obvious consequence of our theorem we have the following result.

COROLlaRY 4. Let $f$ be multianalytic at a point $z_{0}$, finite or infinite. If $z_{0}$ is an essential singularity of $f$, then in every annular neighborhood of $z_{0}$ the values of $f$ are dense in the extended complex plane.

It is worthwhile noting that Corollary 4 follows quite easily from Corollaries 1 and 3.

\section{REFERENCES}

1. M. B. Balk, Picard's theorem for bianalytic entire functions, Dokl. Akad. Nauk SSSR, 152 (1963), 1282 = Soviet Math. Dokl., 4 (1963), 1529.

2. - The big Picard theorem for entire bianalytic functions, Uspehi Mat. Nauk, 20 (1965), No. 2, 159-165.

3. - On the values taken by entire polyanalytic functions, Dokl. Akad. Nauk SSSR, 167 (1966), 12-15 = Soviet Math. Dokl., 7 (1966), 308-311.

4. W. Bosch and P. Krajkiewicz, The big Picard theorem for polyanalytic functions, Proc. Amer. Math. Soc., 26 (1970), 145-150.

5. W. K. Hayman, Meromorphic Functions, Oxford Math. Monographs, Clarendon Press, Oxford, 1964.

6. P. Montel, Leçons sur les Familles Normales de Fonctions Analytiques et Leurs Applications, Gauthier-Villars, Paris, 1927.

7. W. Saxer, Uber eine Verollgemeinerung des Satzes von Schottky, Composition Math., 1 (1934), 207-216.

Received June 30, 1972 and in revised form December 20, 1972.

UNIVERSITY OF NEBRASKA 



\section{PACIFIC JOURNAL OF MATHEMATICS}

\section{EDITORS}

RICHARD ARENS (Managing Editor)

University of California

Los Angeles, California 90024

\author{
R. A. Beaumont \\ University of Washington \\ Seattle, Washington 98105
}

J. Dugundu*

Department of Mathematics

University of Southern California

Los Angeles, California 90007

D. Gilbarg and J. Milgram

Stanford University

Stanford, California 94305

\section{ASSOCIATE EDITORS}
E. F. BECKENBACH
B. H. NeumanN
F. WOLF
K. YosHIDA

\section{SUPPORTING INSTITUTIONS}

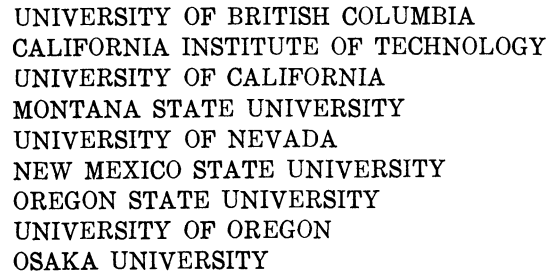

UNIVERSITY OF BRITISH COLUMBIA CALIFORNIA INSTITUTE OF TECHNOLOGY

UNIVERSITY OF CALIFORNIA

MONTANA STATE UNIVERSITY

UNIVERSITY OF NEVADA

NEW MEXICO STATE UNIVERSITY

OREGON STATE UNIVERSITY

UNIVERSITY OF OREGON

OSAKA UNIVERSITY

\author{
UNIVERSITY OF SOUTHERN CALIFORNIA \\ STANFORD UNIVERSITY \\ UNIVERSITY OF TOKYO \\ UNIVERSITY OF UTAH \\ WASHINGTON STATE UNIVERSITY \\ UNIVERSITY OF WASHINGTON \\ * * * \\ AMERICAN MATHEMATICAL SOCIETY \\ NAVAL WEAPONS CENTER
}

The Supporting Institutions listed above contribute to the cost of publication of this Journal, but they are not owners or publishers and have no responsibility for its content or policies.

Mathematical papers intended for publication in the Pacific Journal of Mathematics should be in typed form or offset-reproduced, (not dittoed), double spaced with large margins. Underline Greek letters in red, German in green, and script in blue. The first paragraph or two must be capable of being used separately as a synopsis of the entire paper. Items of the bibliography should not be cited there unless absolutely necessary, in which case they must be identified by author and Journal, rather than by item number. Manuscripts, in duplicate if possible, may be sent to any one of the four editors. Please classify according to the scheme of Math. Rev. Index to Vol. 39. All other communications to the editors should be addressed to the managing editor, or Elaine Barth, University of California, Los Angeles, California, 90024.

50 reprints are provided free for each article; additional copies may be obtained at cost in multiples of 50 .

The Pacific Journal of Mathematics is issued monthly as of January 1966. Regular subscription rate: $\$ 48.00$ a year (6 Vols., 12 issues). Special rate: $\$ 24.00$ a year to individual members of supporting institutions.

Subscriptions, orders for back numbers, and changes of address should be sent to Pacific Journal of Mathematics, 103 Highland Boulevard, Berkeley, California, 94708.

PUBLISHED BY PACIFIC JOURNAL OF MATHEMATICS, A NON-PROFIT CORPORATION

Printed at Kokusai Bunken Insatsusha (International Academic Printing Co., Ltd.), 270, 3-chome Totsuka-cho. Shinjuku-ku, Tokyo 160, Japan.

* C. R. DePrima California Institute of Technology, Pasadena, CA 91109, will replace J. Dugundji until August 1974.

Copyright (C) 1973 by

Pacific Journal of Mathematics

All Rights Reserved 


\section{Pacific Journal of Mathematics}

Vol. 48, No. $2 \quad$ April, 1973

Mir Maswood Ali, Content of the frustum of a simplex................

Mieczyslaw Altman, Contractors, approximate identities and factorization

in Banach algebras ................................ 323

Charles Francis Amelin, A numerical range for two linear operators ...... 335

John Robert Baxter and Rafael Van Severen Chacon, Nonlinear functionals

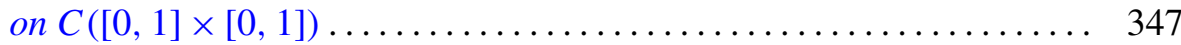

Stephen Dale Bronn, Cotorsion theories....................... 355

Peter A. Fowler, Capacity theory in Banach spaces............... 365

Jerome A. Goldstein, Groups of isometries on Orlicz spaces ........... 387

Kenneth R. Goodearl, Idealizers and nonsingular rings . ............ 395

Robert L. Griess, Jr., Automorphisms of extra special groups and

nonvanishing degree 2 cohomology ..................... 403

Paul M. Krajkiewicz, The Picard theorem for multianalytic functions . . . . 423

Peter A. McCoy, Value distribution of linear combinations of axisymmetric harmonic polynomials and their derivatives ...................

A. P. Morse and Donald Chesley Pfaff, Separative relations for

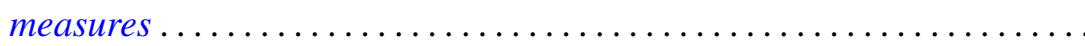

Albert David Polimeni, Groups in which $\operatorname{Aut}(G)$ is transitive on the

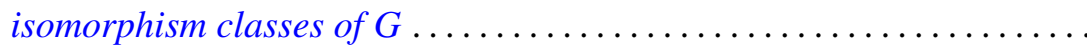

Aribindi Satyanarayan Rao, Matrix summability of a class of derived

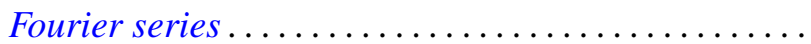

Thomas Jay Sanders, Shape groups and products

Ruth Silverman, Decomposition of plane convex sets. II. Sets associated

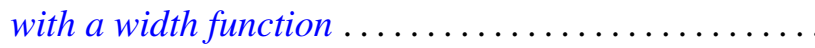

Richard Snay, Decompositions of $E^{3}$ into points and countably many flexible dendrites.............................

John Griggs Thompson, Nonsolvable finite groups all of whose local subgroups are solvable, IV ...

Robert E. Waterman, Invariant subspaces, similarity and isometric equivalence of certain commuting operators in $L_{p} \ldots$

James Chin-Sze Wong, An ergodic property of locally compact amenable

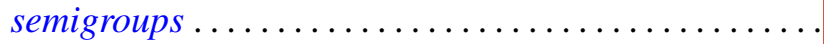

Julius Martin Zelmanowitz, Orders in simple Artinian rings are strongly equivalent to matrix rings ....................... 